\title{
Bifidobacterium longum subsp infantis CECT7210-supplemented formula reduces diarrhea in healthy infants: a randomized controlled trial
}

\author{
Joaquin Escribano', Natalia Ferrée ${ }^{2}$, Mariona Gispert-Llaurado ${ }^{2}$, Veronica Luque ${ }^{2}$, Carmen Rubio-Torrents ${ }^{2}$, \\ Marta Zaragoza-Jordana ${ }^{2}$, Isabel Polanco ${ }^{3}$, Francisco M Codoñer ${ }^{4}$, Empar Chenoll ${ }^{5}$, Mireia Morera ${ }^{6}$, \\ Jose Antonio Moreno-Muñoz ${ }^{4}$, Montserrat Rivero ${ }^{6}$ and Ricardo Closa-Monasterolo ${ }^{7}$
}

BACKGROUND: Intestinal microbiota of breast-fed infants is plenty of beneficial bifidobacteria. We aimed to determine whether an infant formula supplemented with probiotic Bifidobacterium longum subsp. infantis CECT7210 (B. infantis IM1) is effective at reducing diarrhea incidence in healthy term infants.

METHODS: Double-blinded, randomized, multicenter, controlled clinical trial, where formula-fed infants ( $<3$ months) received an infant formula supplemented (Probiotic) or not (Control) with $10^{7} \mathrm{cfu} / \mathrm{g}$ of $B$. infantis IM1 over 12 weeks. Diarrheas, growth, digestive symptoms, stool bifidobacteria, and microbiota were assessed.

RESULTS: In all, 97 (Control) and 93 (Probiotic) infants were randomized, and 78 (Control) and 73 (Probiotic) completed the 12 week-follow-up. In the overall study period, a median of $0.29 \pm 1.07$ and $0.05 \pm 0.28$ diarrhea events/infant was observed in the Control and Probiotic groups, respectively $(P=0.059)$. This trend to less diarrhea episodes in the Probiotic group reached statistical significance at 8 weeks $(0.12 \pm 0.47$ vs. $0.0 \pm 0.0$ events/infant, $P=0.047)$. Constipation incidence was higher (odds ratio (OR) $2.67(1.09-6.50)$ ) and stool frequency lower $(2.0 \pm 1.0$ vs. $2.6 \pm 1.3$ stools/day, $P=0.038)$ in the Control group after 4 weeks. No differences were found at other time points nor in other digestive symptoms, growth, or formula intake.

CONCLUSION: A B. infantis IM1-supplemented infant formula may reduce diarrhea episodes, being safe, well tolerated, and associated with lower constipation prevalence.

T here is compelling evidence that infant nutrition has a great impact on the bowel microbiota balance and also on the beneficial effects of Lactobacillus and Bifidobacterium genera $(1,2)$. The microbiota modulates the development of intestinal immunity, and previous studies demonstrated that an increased presence of both Bifidobacterium and
Lactobacillus is associated with a lower incidence of gastrointestinal infections $(3,4)$.

Breast-fed infants' intestinal microbiota includes higher proportions of Bifidobacterium and Lactobacillus than those of formula-fed infants, who have a less diverse microbiota and an increased number of pathogens, such as Bacteroides, Enterobacteriaceae, and Clostridium (5). These differences have been associated with higher rates of infectious and noninfectious diseases in the latter, suggesting that breast-fed infants are better protected against gastrointestinal infections than formula-fed infants. Consistently, Lara-Villosada et al. (6) demonstrated an association between higher amount of Lactobacillus and lower incidence of diarrhea in breast-fed infants.

Formula manufacturers aim to offer infant formulas that partially mimic human milk benefits (7), for example, adding probiotics.

Previous studies and a systematic review by the ESPGHAN Committee on Nutrition concluded that probiotic supplementation of infant formula has no adverse effects on infant growth $(8,9)$ and may produce benefits, such as the prevention of infections $(10,11)$, allergic disorders (12), and inflammatory bowel intestinal disease in older children (13). Regarding gastrointestinal infections, some studies have reported the benefits of probiotics in reducing their incidence and severity $(11,14)$. However, as these observations were inconclusive, the ESPGHAN Committee on Nutrition found that further studies were necessary (15). Moreover, the ESPGHAN Working group on Probiotics and Prebiotics showed that the effects of probiotics were strain-specific, and, thus, the efficacy, safety, as well as recommendations for use should be established of each microorganism (16).

According to these results, the Bifidobacterium longum subsp. infantis CECT7210 (B. infantis IM1), a new probiotic strain isolated from breast-fed infant feces, has been shown in vitro to inhibit rotavirus replication and to protect against

\footnotetext{
${ }^{1}$ Paediatrics Unit, Hospital Universitari Sant Joan de Reus, Institut d'Investigació Sanitaria Pere Virgili, Reus, Spain; ${ }^{2}$ Paediatrics Research Unit, Universitat Rovira I Virgili, Institut d'Investigació Sanitaria Pere Virgili, Reus, Spain; ${ }^{3}$ Department of Paediatric Gastroenterology and Nutrition, La Paz University Hospital, Madrid, Spain; ${ }^{4}$ Lifesequencing SL, Valencia, Spain; ${ }^{5}$ Biopolis SL, Valencia, Spain; ${ }^{6}$ Laboratorios ORDESA SL, Barcelona, Spain; ${ }^{7}$ Neonatal Unit, Hospital Universitari de Tarragona Joan XXIII, Institut d'Investigació Sanitaria Pere Virgili, Tarragona, Spain. Correspondence: Ricardo Closa-Monasterolo (ricardo.closa@urv.cat)

Received 29 August 2017; revised 23 January 2018; accepted 8 February 2018; advance online publication 2 May 2018. doi:10.1038/pr.2018.34
} 
virus infection in animal models (17). This result, in addition to previous data about probiotic use, led us to hypothesize that $B$. infantis IM1 could have an impact on immune function by improving both specific biomarkers and infection incidence. Our aim is to determine whether an infant formula supplemented with this probiotic is effective at reducing the incidence of diarrhea in healthy term infants.

\section{METHODS}

\section{Study Design}

The study was designed, following the CONSORT statement (www. consort-statement.org), as a multicenter, double-blind, randomized, placebo-controlled parallel group trial, where formula-fed healthy term infants ( $<3$ months) received an infant formula supplemented with $10^{7} \mathrm{cfu} / \mathrm{g}$ of $B$. infantis IM1 (Probiotic) or a standard formula (Control) during 12 weeks with a 1:1 allocation ratio. Study visits were performed at 4,8 , and 12 weeks of intervention. The research staff was blinded to both treatment allocation and data analysis. In addition, participants and caregivers were blinded throughout the study. No changes to methods after trial commencement were included.

\section{Participants}

Subjects were recruited at the Hospital Infantil La Paz de Madrid, the Hospital Universitari de Tarragona Joan XXIII, and the Hospital Universitari Sant Joan de Reus (Spain). Seven different investigators contacted families at the Hospitals, before the delivery discharge, to explain the study only if their infants were exclusively formula-fed. The same investigators performed visits at the Hospitals. The inclusion criteria were as follows: to be healthy-term newborns ( $\geq 37$ weeks), with normal birth weight (3rd to 97th percentiles for gestational age), normal growth curve according to current Spanish references (18), and $\leq 3$ months of age at enrolment. Moreover, exclusively formula-fed at enrolment, with $\leq 30$ days of breastfeeding (stopped at least 15 days before inclusion), $\leq 48 \mathrm{~h}$ of feeding with pre- or probiotic-supplemented infant formulas (with a washout period of 7 days with control formula). Families agreed to exclude any source of pre- or probiotics during the study. The exclusion criteria were as follows: congenital illness or malformations that may affect infant feeding and/or growth, significant pre- or postnatal diseases, family history of atopy, any pathology related with the immune or gastrointestinal system, suspected or known allergy to cow's milk protein, and receiving pre- or probiotics within less than 15 days before the enrolment.

\section{Study Intervention}

Infants received an infant formula supplemented with $B$. infantis IM1 in a dose of $10^{7} \mathrm{cfu} / \mathrm{g}$ (Probiotic) or without supplementation (Control; Supplementary Table S1 online). Laboratorios Ordesa SL (Spain) produced both formulas, in compliance with current EU standards (Commission Directive 2006/141/EC).

Two codes for each study formula were used. The random allocation sequence was generated by ORDESA SL (by software in blocs of eight patients) and was provided to a study member not involved in recruitment or study visits. Preparation instructions were given to avoid excessive heating of the study formula. Intake of any pre- or probiotic-supplemented product or intake of other formulas over more than 10 days was a study discontinuation criteria and was controlled through a food intake questionnaire.

\section{Study Outputs}

The primary outputs for this randomized clinical trial (RCT) were diarrhea incidence, growth, and digestive tolerance. Secondary outputs were other infections, immune system response, and microbiota analysis.
Infection incidence. Clinical data were recorded at recruitment, 4, 8, and 12 weeks. A complete examination was performed at recruitment by a pediatrician to detect any physical or system (respiratory, oropharyngeal, ophthalmic, and other) anomaly. Infections were assessed in each study visit using a diary record validated by the pediatrician. The record included the presence/ absence (yes/no) and number of events of diarrhea described as three or more liquid stools in $24 \mathrm{~h}$ (in infants who do not usually fulfil this deposition pattern), vomiting, respiratory infection (upper and lower tracts), otitis, conjunctivitis, and urinary tract infections reported by the parents and confirmed by the pediatrician by assessing the clinical symptoms and prescribed medication. The use of any medication was reported in a concomitant medication diary record completed by parents with the physician's advice.

Growth and digestive tolerance. Anthropometry was assessed at recruitment, 4,8 , and 12 weeks ( \pm 3 days). The nude weight and length of the infant were determined using a SECA 336 scale (SECA, Hamburg, Germany; precision: $\pm 0.05 \mathrm{Kg}$ ) and a SECA 232 stadiometer (precision: $\pm 1 \mathrm{~mm}$ ), and the BMI was calculated. Head circumference was determined using a SECA non-extendable insertion tape (precision: $1 \mathrm{~mm}$ ). $Z$-scores for anthropometric measurements were calculated according to the World Health Organization (WHO) Standards using the WHO software (19). Parents recorded during 3 days before each visit the presence/ absence of digestive symptoms (flatulence, abdominal pain, constipation (defined as less than one hard stool/day or less than one stool/2 days), and clinical symptoms compatible with the i8486325194nfant colic (defined as incontrollable crying for more than $3 \mathrm{~h} /$ day, more than 3 days/week, and more than 3 weeks)). The stool frequency (stools/day), stool consistency (according to the Modified Bristol Stool Form Scale for children adapted to types 1-3), vomiting ( $n /$ day), and regurgitation (from 0 to 5 , from 5 to 15 , or $>15$ events/day) were also recorded. Finally, crying (h/day), sleeping behaviors (sleeping hours and breaks during the night and totals), and the presence of diaper dermatitis were also recorded. Any other adverse event was recorded and classified as mild, moderate, or severe.

Feeding data (days of previous breastfeeding, age at formula introduction, type, and total volume intake of formula per day $(\mathrm{ml} /$ day)) were recorded at recruitment. During follow-up, total volume intake of study formula per day and the use of other formulas were recorded.

Immune system response. Saliva samples were obtained at recruitment and after 12 weeks of intervention. We used specific swabs for saliva collection (Sarstedt, Nümbrecht, Germany). A minimum of $0.5 \mathrm{ml}$ of saliva was needed to perform posterior analyses. When insufficient baseline sample was obtained, the procedure was repeated at posterior follow-up visits. Secreted immunoglobulin A (IgA; mg/dl) was measured using a commercial enzyme-linked immunosorbent assay (ELISA) following the manufacturer's instructions (Bethyl Laboratories; Montgomery). After the ELISA for IgA detection, a second ELISA was performed to confirm that the detected IgA concentration corresponded to the secretory fraction by a monoclonal mouse antihuman secretory component antibody (HyTest Laboratories; Turku, Finland) and the ELISA components used previously.

Rotavirus-specific IgA was also analyzed in the saliva samples by an in-house-developed ELISA method as previously described by Colomina et al. (20).

All the vaccinations received by the infant throughout the study period were recorded.

Microbiota analysis in stool samples. Stool samples were obtained within the 2 days before study visits, frozen, and transported to the study centers with ice pads. The samples were preserved at $-80^{\circ} \mathrm{C}$ until they were shipped to a central laboratory (Biopolis SL, Valencia, Spain) for their analysis. 


\section{Articles | Escribano et al.}

Total amount of Bifidobacterium and B. infantis IM1 were determined by real-time PCR using specific primers for genus (21), and specific primers and probe for $B$. infantis IM1 (with a specificity greater than $95 \%$ (data not shown; details are described in Supplementary Table S2 online). Total DNA was extracted from feces using "QIAamp DNA stool mini kit" (Qiagen, Barcelona, Spain) following the manufacturers' manual. A microbiota analysis by massive genome sequencing was performed in a subsample of infants (25 and 26 from Probiotic and Control groups, respectively) using 454 platform (Roche Life Science, Basel, Switzerland). DNA was isolated following Yuan et al. (22) with minor modifications by MagnaPure Compact System (Roche Life Science). Libraries were built following Roche recommendations and using Sim and coworkers set of primers (23), specially modified in order to optimize Bifidobacterium detection. Sequencing was performed using 454 platform under pattern B flow, following the manufacturer's instructions. The resulting sequences for each patient were treated for quality and the clean sequences were used for taxonomical association using QIIME (24).

\section{Ethics}

The study was preformed following the Helsinki declaration and the guidelines for the ethical conduct of medical research involving children, and was approved by the local Ethical Committees involved. All parents received written information and signed informed consent. The trial was registered at Clinicaltrials.gov as NCT02096302 (https://clinicaltrials.gov/ct2/show/NCT02096302? term $=$ NCT02096302.\&rank $=1$ ).

\section{Statistics}

Quantitative data were presented as the mean \pm SD. Categorical variables were presented as absolute and relative frequencies $(n, \%)$.

We performed a cross-sectional analysis comparing both study groups at individual time points (at recruitment, 4, 8, and 12 weeks) as well as a longitudinal analysis comparing changes throughout the study. Cross-sectional differences in quantitative variables were analyzed by Student's $t$-test or Mann-Whitney $U$-test as appropriate. Frequencies of categorical variables were analyzed by $\chi^{2}$-test. When possible, odds ratio (OR; 95\% confidence interval) for the association between categorical variables was calculated. The longitudinal analysis was performed by two-way ANOVA for repeated measures. In this model, time points (recruitment, 4, 8, and 12 weeks) and group (Control vs. Probiotic) were analyzed together as the two factors influencing the outcomes. The $P$ value for the interaction between both factors showed the probability of a different longitudinal evolution between groups. Multivariate analysis was performed to evaluate the combined effect of $B$. infantis IM1 and vaccinations on the salivary concentration of IgA using a linear regression model. Statistical significance was accepted at the level of 0.05. We used IBM SPSS Statistics for Windows, Version 20.0 (Armonk, NY, IBM). The sample size (93 per group) was calculated, using data reported by Weizman et al. (25) to detect a difference between groups of $\sim 0.033$ events/infant in diarrhea episodes $(1 / 3$ of the SD in the control group). We used the Epidat 3.1 program (available at: http://www.paho.org/spanish/sha/epidat.htm), an $\alpha$ error of 0.05 , a power of $80 \%$, and assumed an attrition rate of $20 \%$. R version 3.1 .3 was used to assess alpha and beta diversity in the microbiome analysis, as well as to compare different parameters under Wilcoxon non-parametric test.

\section{RESULTS}

\section{Study Sample and Recruitment Characteristics}

From January 2011 to August 2012, 194 eligible infants were recruited. Among those, four were excluded because of previously undetected exclusion criteria (three cases) or consent removal. One hundred ninety infants finally started the study (93 and 97 in Probiotic and Control groups, respectively), from which one hundred fifty-one (73 and 78 in Probiotic and Control groups, respectively) completed the 12 weeks of intervention (Figure 1). The distribution of infants into the study groups was similar in all the participating centers $(P=0.764)$. The mean age at recruitment was $7.3 \pm 13.3$ days (with a median (IQR) of $3(2,4)$ days and a range between 1 and 56 days or 0 and 83 days for Probiotic and Control groups, respectively).

Baseline characteristics, such as gender distribution, age at recruitment, anthropometry at birth and at recruitment (Figure 2a), type of delivery, number of siblings, formula intake, and digestive symptoms were similar among groups (Table 1). Incidence of infant colic was higher in the Control group $(9.5 \%$ vs. $2.2 \%, P=0.034$; Table 1$)$. There were no differences in the physical and system infants' examinations between groups (data not shown).

\section{Effect of Intervention on Infections}

The incidence of diarrhea was very low, as only seven Control and three Probiotic infants had events throughout the study period. Although percentage of infants who suffered diarrhea did not show statistically significant differences between study groups (4.1\% in Probiotics vs. 9.0\% in Control; $P=0.230$; OR $0.44(0.11-1.75))$, the number of episodes was significantly higher in the Control group (23 events compared with 4 in the Probiotic group). In the overall study period, infants in the Control group showed a median of $0.29 \pm 1.07$ events/infant, whereas those in the Probiotic showed $0.05 \pm 0.28$ events/infant $(P=0.059)$. This lower prevalence of diarrhea episodes among the Probiotic group was significant at 8 weeks of intervention $(0.12 \pm 0.47$ events/infant vs. $0 \pm 0$ events/infant for the Control and Probiotic groups, respectively; $P=0.047$ ) but did not reach statistical significance at the other time points (Figure 3 ). The incidence of other infections was not significantly different between the study groups. A total of $33.3 \%$ of infants in the Control group and $38.5 \%$ in the Probiotic had respiratory infections $(P=0.520)$ throughout the study period. The other assessed infections had a lower incidence (1.4\%, 1.4\%, and $4.2 \%$ for otitis, urinary tract infection, and conjunctivitis, respectively) and were equally distributed between the study groups. Concomitant medication diaries showed that $74.6 \%$ of the infants did not receive any medication during the study. Oppositely, seven infants in total received oral antibiotic therapy for any adverse event. Those infants correspond to $3.6 \%$ of the sample without differences in distribution between groups (four in Control group and three in Probiotic, $P=0.268)$. Moreover, we identified 21 infants whose parents reported the use of medication but we fail to assess the type of medication due to incomplete information in the concomitant medication diary. Those infants correspond to $11 \%$ of the sample and were equally distributed between groups (13 in Control group and 8 in the Probiotic group, $P=0.307)$.

\section{Effect of Intervention on Infant Growth, Digestive Symptoms, and Formula Intake}

There were no significant differences between groups in the $z$-scores of anthropometric data throughout the study period, 


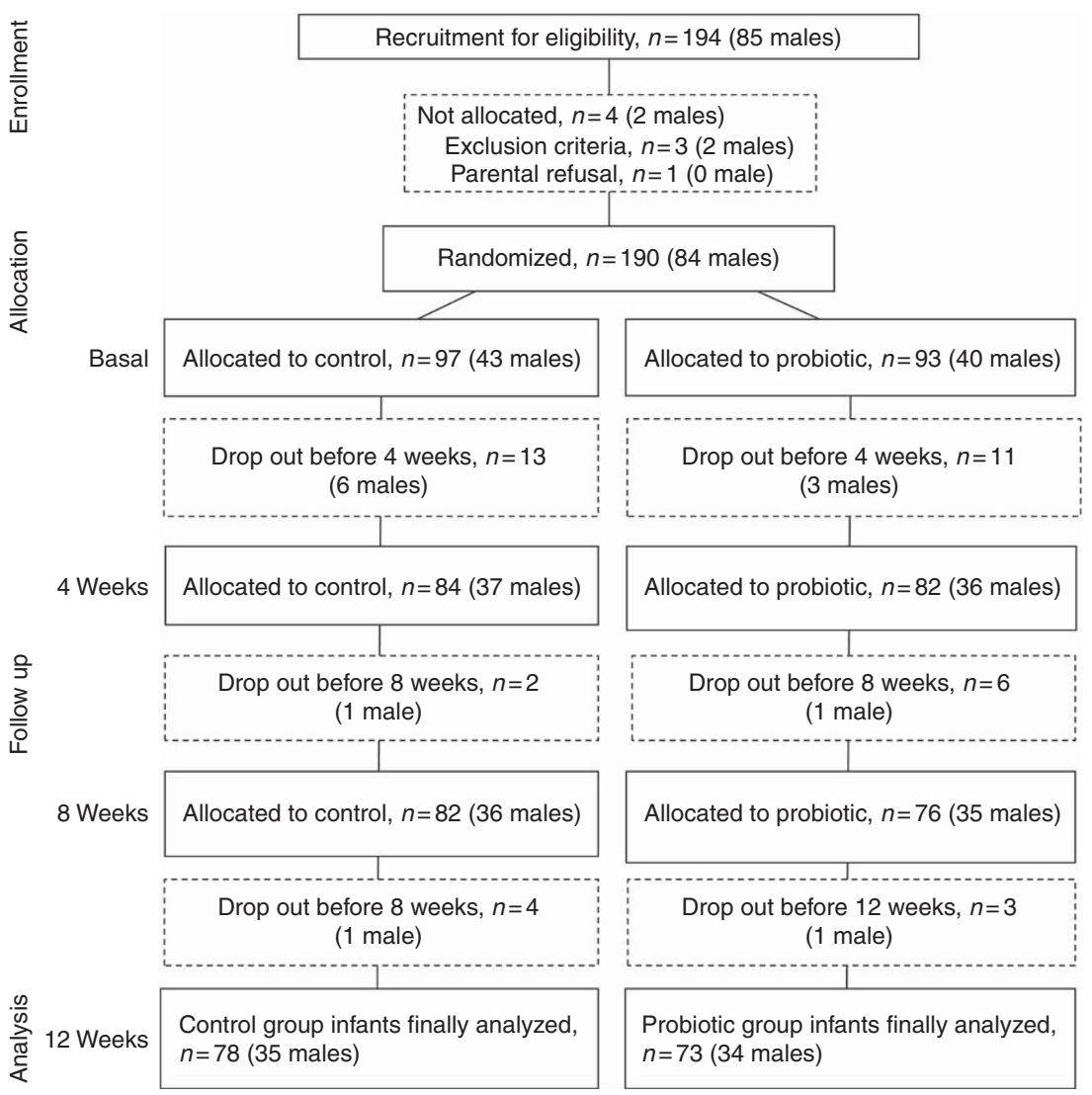

Figure 1. Flowchart of recruitment, randomization, follow-up, and withdrawals at each study time point.
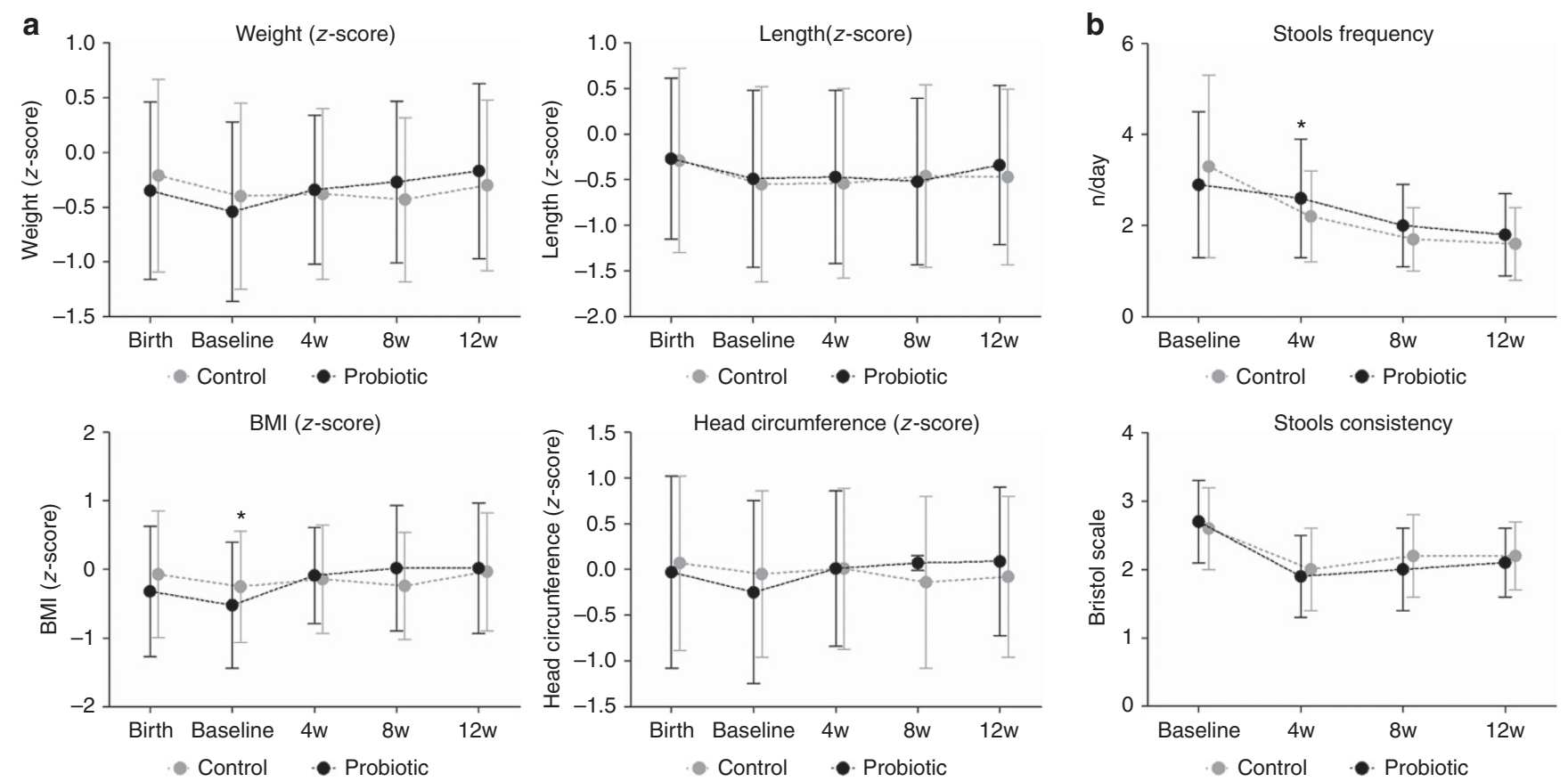

Figure 2. Safety and efficacy parameters. Anthropometric characteristics (a) and stool frequency and consistency (b) of infants by feeding group at each study time point. $W$, weeks. ${ }^{*} P<0.05$. 


\section{Articles | Escribano et al.}

Table 1. Baseline characteristics of infants

\begin{tabular}{|c|c|c|c|}
\hline & Control group (mean \pm SD) or (\%) $n=97$ & Probiotic group (mean \pm SD) or (\%) $n=93$ & $P$ value \\
\hline Age at recruitment (days) & $8.6 \pm 16.3$ & $5.9 \pm 9.2$ & 0.500 \\
\hline Gender (\% males) & 44.3 & 43.5 & 0.906 \\
\hline Gestational age (weeks) & $39.2 \pm 1.1$ & $39.3 \pm 1.2$ & 0.802 \\
\hline Mother's age (years) & $31.9 \pm 4.7$ & $32.6 \pm 5.1$ & 0.288 \\
\hline Perinatal complications ( $\%$ yes) & 4.2 & 5.4 & 0.684 \\
\hline Delivery type (\% caesarean) & 31.2 & 26.1 & 0.209 \\
\hline Siblings $(n)$ & $0.7 \pm 0.7$ & $0.8 \pm 1.0$ & 0.930 \\
\hline Mean intake volume (ml/day) & $210.0 \pm 237.7$ & $190.7 \pm 207.8$ & 0.500 \\
\hline Stool frequency ( $n /$ day) & $3.3 \pm 2.0$ & $2.9 \pm 1.6$ & 0.183 \\
\hline Vomits (n/day) & $0.1 \pm 0.4$ & $0.2 \pm 0.6$ & 0.411 \\
\hline Regurgitation (n/day) & $1.1 \pm 0.4$ & $1.1 \pm 0.3$ & 0.653 \\
\hline Irritability (\% yes) & 12.8 & 13.0 & 1.000 \\
\hline Flatulence (\% yes) & 22.3 & 18.5 & 0.587 \\
\hline Abdominal pain ( $\%$ yes) & 17.9 & 8.7 & 0.085 \\
\hline Colics (\% yes) & 9.5 & 2.2 & 0.034 \\
\hline Constipation (\% yes) & 6.3 & 6.5 & 1.000 \\
\hline
\end{tabular}
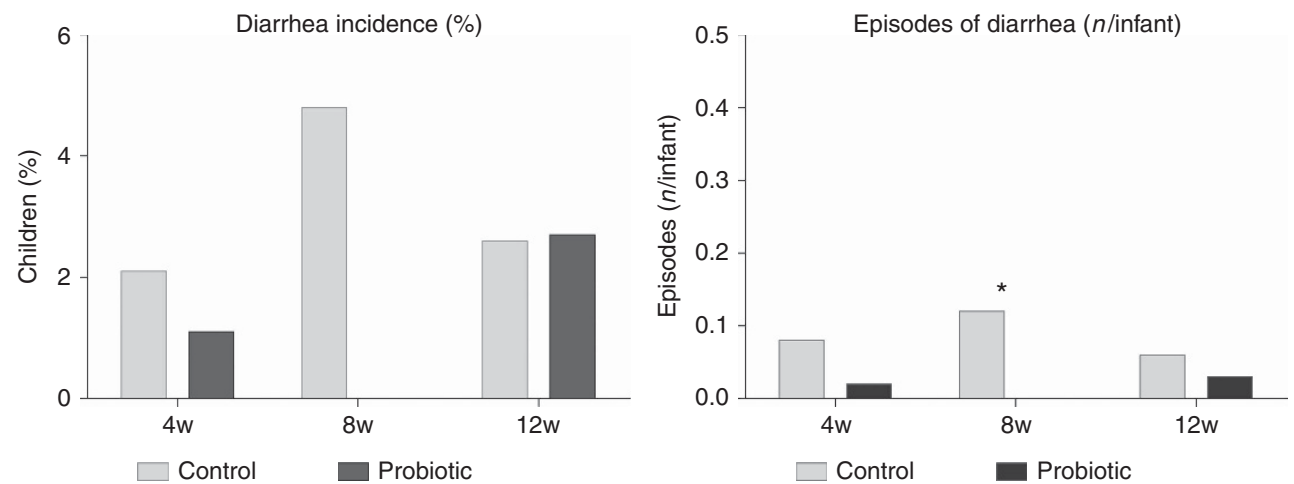

Figure 3. Infants with diarrhea episodes and number of episodes throughout the study period by feeding group.

and there were no cross-sectional differences upon longitudinal analyses (Figure 2a). The infants in the Probiotic group had a lower BMI at recruitment compared with those in the Control group, but showed similar results at other time points (Figure 2).

Deposition frequency was higher in the Probiotic group compared with Control (at 4 weeks; $P=0.038$; Figure 2b). Moreover, longitudinal analysis showed that the drop in stool frequency observed throughout the study follow-up in both groups was lower in the Probiotic group than in the Control $(P=0.046)$. Accordingly, the percentage of infants who reported constipation was lower in the Probiotic group (22.6\% vs. $9.9 \%$ at 4 weeks $(P=0.027)$ and $13 \%$ vs. $2.8 \%$ at 12 weeks of intervention $(P=0.033)$ for the Control and Probiotic groups, respectively). The OR for suffering constipation in infants from the Control group compared with the Probiotic was $2.67(1.09-6.50)$ and $5.22(1.10-24.73)$ at 4 and 12 weeks, respectively. No differences between groups were observed in the usual stool consistency throughout the study (Figure $2 b$ ).

There were no differences between groups in terms of digestive symptoms, behavior, or total daily formula intake throughout the study period (Table 2). We found a significant difference in sleeping hours at night at 8 weeks of follow-up (Table 2). There were no significant differences in the presence of adverse events between study groups nor in the absolute number score of the severity of the adverse event (data not shown).

\section{Effect of Intervention on the Microbiota in the Faeces and Immunological Parameters}

Real-time PCR results showed no differences in total Bifidobacterium levels in the feces between groups. However, as expected, there was a significant increase in B. infantis IM1 counts in the Probiotic group. These differences appeared after 4 weeks of intervention and were maintained throughout 
Table 2. Frequency of digestive symptoms (vomiting, regurgitation, flatulence, and abdominal pain) and behavior as well as formula intake throughout the study period

\begin{tabular}{lccc}
\hline & $\begin{array}{c}\text { Control group, } \\
n=95\end{array}$ & $\begin{array}{c}\text { Probiotic group, } \\
n=93\end{array}$ & $P$ value \\
\hline Vomits (\% children with one or more than one vomit/day) & \\
Baseline evaluation & 9.5 & 11.9 & 0.492 \\
At 4 weeks & 12.3 & 11.3 & 0.587 \\
At 8 weeks & 8.2 & 6.1 & 1.000 \\
At 12 weeks & 6.3 & 6.0 & 0.854 \\
& & \\
Regurgitation (\% children with more than five regurgitation/day) \\
Baseline evaluation & 10.7 & 12.9 & 0.570 \\
At 4 weeks & 13.1 & 14.8 & 0.908 \\
At 8 weeks & 11.0 & 9.3 & 0.734 \\
At 12 weeks & 5.2 & 9.7 & 0.291
\end{tabular}

Flatulence (\% children with flatulence)

$\begin{array}{llll}\text { Baseline evaluation } & 22.3 & 18.5 & 0.514 \\ \text { At } 4 \text { weeks } & 54.3 & 50.0 & 0.588 \\ \text { At } 8 \text { weeks } & 47.2 & 47.9 & 0.937 \\ \text { At } 12 \text { weeks } & 35.1 & 28.2 & 0.368\end{array}$

Abdominal pain (\% children with pain)

Baseline evaluation $\quad 17.9$

Sleeping breaks at night (\% of yes)

$\begin{array}{lrrl}\text { Baseline evaluation } & 12.6 & 13.2 & 1.000 \\ \text { At } 4 \text { weeks } & 18.3 & 20.3 & 0.842 \\ \text { At } 8 \text { weeks } & 9.1 & 19.4 & 0.098 \\ \text { At } 12 \text { weeks } & 6.7 & 6.9 & 1.000\end{array}$

Sleeping hours at night (mean $\pm S D$ )

$\begin{array}{llll}\text { Baseline evaluation } & 7.4 \pm 1.9 & 7.5 \pm 1.6 & 0.858 \\ \text { At } 4 \text { weeks } & 7.1 \pm 1.6 & 6.8 \pm 1.4 & 0.271 \\ \text { At } 8 \text { weeks } & 8.3 \pm 1.6 & 7.4 \pm 1.5 & 0.012 \\ \text { At } 12 \text { weeks } & 8.3 \pm 1.4 & 8.0 \pm 1.5 & 0.260\end{array}$

Formula intake (ml/day, median $\pm S D)$

$\begin{array}{llll}\text { At } 4 \text { weeks } & 816 \pm 153 & 801 \pm 149 & 0.513 \\ \text { At } 8 \text { weeks } & 856 \pm 152 & 825 \pm 189 & 0.255 \\ \text { At } 12 \text { weeks } & 890 \pm 169 & 851 \pm 145 & 0.133\end{array}$

the study period (Figure 4a). This effect was reproduced in the microbiota analysis, where an increase in B. longum was detected at the final point in the Probiotic group $(P=0.023)$, that was not observed in the Control $(P=0.45$; Figure $4 b)$.
Microbiota analysis pinpoints a nonsignificant decrease in the presence of pathogens (Escherichia, Clostridium, Salmonella, Campilobacter, and Yersinia) in the Probiotic group at 12 weeks compared with those taking the Control formula, which did show equal values than at baseline. We also observed in the Control group a significant increase in Escherichia coli at final time point with respect to baseline, whereas the presence of the pathogen did not change along the follow-up in the Probiotic group (Supplementary Figure S3 online).

In all, $86.3 \%$ of the infants in our sample were vaccinated at least once during the study period, with no differences in distribution between groups. A $34.2 \%$ of the participants was vaccinated against rotavirus (one or two doses), also equally distributed between study groups.

Regarding immune markers, both groups showed a nonsignificant tendency toward an increase in IgA concentrations throughout the study. We also found similar concentrations of $\operatorname{IgA}$ among groups $(1.24 \pm 1.99$ vs. $0.78 \pm 1.10 \mathrm{mg} / \mathrm{dl}, P=0.739$ at recruitment and $2.26 \pm 2.34$ vs. $2.66 \pm 3.85 \mathrm{mg} / \mathrm{dl}, P=0.803$ at 12 weeks of intervention; Control and Probiotic groups, respectively). However, using a linear regression model (adjusted by the number of vaccinations received by the infant (range between 0 and 15) to avoid the effect of this confounding factor) we observed that the presence of $B$. infantis IM1 in the feces modulated IgA concentration at the end of the intervention. The results showed that each $\log _{10} \mathrm{cfu} / \mathrm{g}$ of $B$. infantis IM1 at 4 weeks of intervention increased $\operatorname{IgA}$ concentration by $0.185 \mathrm{mg} / \mathrm{dl}$ (95\% CI: $0.032,0.338 ; P=0.019$ ) at the end of the study. The mean difference of $B$. infantis IM1 in the feces of the Probiotic group infants compared with the Control was $\sim 3$ $\log _{10}$, suggesting a possible effect of a $0.555 \mathrm{mg} / \mathrm{dl}$ of increase in IgAs.

The results of the rotavirus-specific IgA analysis showed a similar percentage of infants with a positive titer of IgAs in both groups (22.0\% vs. $18.5 \%$, for the Control and Probiotic groups at 12 weeks of intervention, respectively; $P=0.648$ ). We also compared the absolute titers, and we did not find differences between the study groups (nor among all infant samples, neither for subgroups of infants (positive titers or vaccinated against rotavirus; data not shown)). The geometric mean titers at 12 weeks of follow-up were 71.2 and 78.8 for the Control and Probiotic groups, respectively.

\section{DISCUSSION}

During the last decade, it is known that the human intestinal microbiota can have a major role in modulating health and disease along lifespan. Thus, efforts have been made to modulate this microbiota through different products such as probiotics, aimed to enrich or selectively stimulate the growth and/or activity of beneficial intestinal bacteria.

This is the first multicenter, double-blind, RCT to demonstrate the safety and efficacy of a B. infantis IM-1supplemented infant formula in healthy infants. Although previous studies evaluated the effect of different probiotics on 

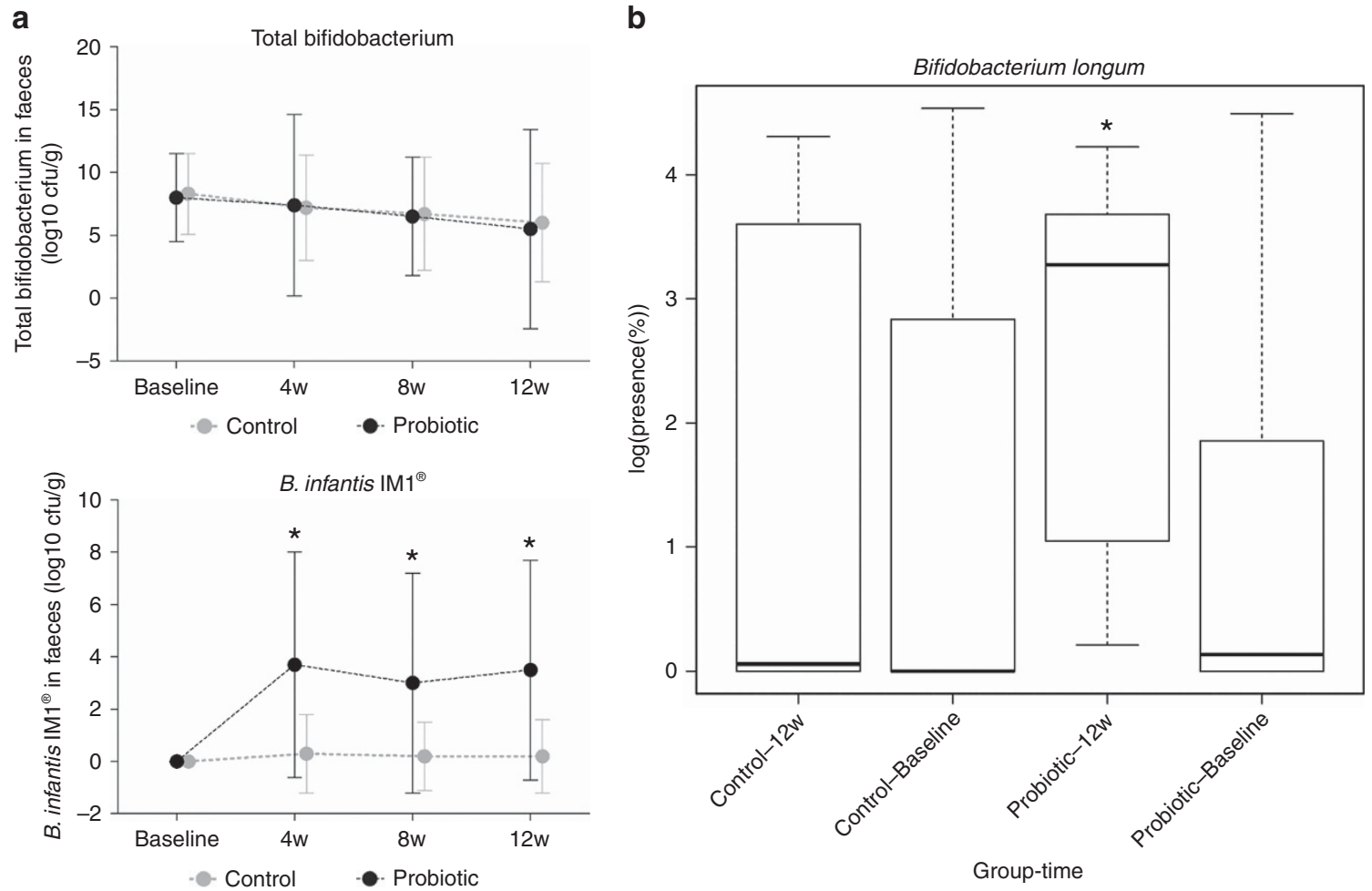

Figure 4. Stool samples analyses. Total Bifidobacterium and B. infantis IM1 detected by RT-PCR in stool samples (a) and B. longum presence detected in the whole microbiota analysis (b). ${ }^{*} P<0.05$ with respect to the baseline.

infants (15), the effect of this specific strain has not been assessed yet.

\section{Efficacy}

The efficacy of the B. infantis IM-1-supplemented formula was demonstrated by the higher presence of the probiotic in stool samples among supplemented infants. In addition, microbiota analysis showed that supplemented formula was associated with constant levels in the Proteobacteria such us E. coli in contrast with the increase observed in the Control group. Similar results were observed in breast-fed infants, as breastmilk prevents the gut colonization by Proteobacteria, potentially reducing the infection risk (5).

The expected increase of the $B$. infantis IM- 1 in the fecal microbiota in the Probiotic group compared with the Control one was not associated with a change in Bifidobacterium concentration. Others have previously observed that the major bacterial group proportions were not influenced by probiotic treatment (26-28). Our results coincide with those previous findings and demonstrate the ability of the supplemented formula to modify the microbiota.

Despite a lower diarrhea episode trend among the Probiotic group, results did not show a sustained effect along the study period, probably because of the small diarrhea incidence among the whole sample. This small incidence of diarrhea was probably due to the infants' young age ( $<3$ months) and the geographic area incidence of gastrointestinal infections. Early in life, infants are under the protective effect of the placenta-transferred maternal antibodies that can protect against the most common germs, and have an effect beyond the first 6 months of life. Moreover, our infants did not attend childcare, what further reduces infection risk. Even in those protected conditions, we found that infants fed probiotic formula had less diarrhea episodes compared with control infants at one of the time points, suggesting a possible effect of probiotics decreasing the incidence of gastrointestinal infections. However, we did not find a significant effect in terms of OR. Previous studies have reported the effect of specific strains of Bifidobacterium on recovery from diarrhea, especially reducing the duration and severity (29). Before our study, Muñoz et al. (17) had already demonstrated the ability of the $B$. infantis IM-1 to inhibit virus infection in an in vitro study as well as to protect against rotavirus infection in a in vivo mouse model. Our results are consistent with those findings, but further studies should find out whether $B$. infantis IM-1 may also inhibit rotavirus infection in healthy infants.

Regarding other infections, the incidence was similar between groups. The lower incidence of those infections throughout the study period may probably support these results. Consistent with our findings, Gibson et al. (30) also found no differences in respiratory infections among supplemented formula-fed infants.

In parallel to the lower incidence of diarrhea episodes in supplemented infants, we found a relationship between the $B$. longum IM-1 levels in the feces and the secretory IgA 
concentration measured at the end of the follow-up. A clear effect of probiotic supplementation was not observed for this immunoglobulin, as concentrations were similar in both groups. However, as the different vaccination patterns in each infant could influence these results, we adjusted the relationship between the $B$. infantis IM- 1 content and the secretory IgA concentration by the number of vaccines received. Results showed that an increase in the probiotic count in the feces was associated with higher IgA concentrations, suggesting that the increase in B. infantis IM-1 is related with the stimulation of the immunological system function.

Previous studies have observed no changes in fecal secretory IgA after Bifidobacterium supplementation (27,31). Holscher et al. (31) found that probiotic supplementation induced a significant increase in secretory IgA only among those infants born by vaginal delivery. Accordingly, we performed the same analyses in vaginally delivered infants ( $71.3 \%$ of our sample), and we observed that the increase in secretory IgA during the study tended to be higher among probiotic-supplemented infants $(P=0.057$, data not shown). Those previous studies analyzed the secretory IgA in the feces, which has a higher concentration of IgA than the saliva (32), and this feature could influence our results.

\section{Safety and Tolerance}

The safety and tolerance of the B. infantis IM-1-supplemented formula were demonstrated as the growth parameters, digestive symptoms, crying behavior, tolerance, and compliance with the study formula were similar compared with the non-supplemented group.

All infants showed similar growth patterns throughout the follow-up. Infants fed probiotic formula showed similar weight, length and head circumference $z$-scores at any time point, as well as similar BMI $z$-scores except at recruitment. Moreover, as observed in previous studies $(30,33,34)$, the evolution of these parameters throughout the study was also similar. Consistent with these results, a systematic review by Mugambi et al. (15) reported no differences in weight, length, and head circumference gain in infants supplemented with different probiotics. Therefore, an ESPGHAN Committee on Nutrition commentary concluded that the supplementation of infant formula with some probiotic strains has no adverse effects on growth in healthy term infants (35). Our results confirm that the $B$. infantis IM- 1 strain is also safe in terms of growth.

Digestive symptoms such as regurgitation, vomiting, and flatulence were similar between both groups during the follow-up period coinciding with Gibson et al., who showed no significant differences in these parameters of digestive tolerance. However, other studies found a lower frequency of regurgitation in probiotic-supplemented formula-fed infants compared with the control ones $(34,36)$.

Constipation is a common adverse effect of feeding infants with a standard formula rather than breastfeeding (37). In our study, infants in the Probiotic group had a higher stool frequency than the Control at 4 weeks of supplementation.
Moreover, longitudinal analysis showed that the expected reduction of the stool frequency during the study was lower in the Probiotic group compared with those observed in the Control. These differences, even that small, had a clinical relevance, as the percentage of infants with constipation at 4 and 12 weeks was lower in the Probiotic group than in the Control. These results are consistent with those from Vendt et al. (38), who observed a higher stool frequency in probiotic-supplemented infants, although most of the studies found no significant differences in the number of bowel movements $(15,30,33)$. Thus far, the evidence-based recommendations developed by ESPGHAN and NASPGHAN in 2014 do not support the use of probiotics in the treatment of childhood constipation (39) and the efficacy must be tested for each specific strain. Regarding the effect of supplemented formula in the stool consistency, we observed no differences between both formula groups similarly to Petschow et al. (40) and Weizman et al. (33). Consistent with this, the most recent systematic review and commentary by the ESPGHAN Committee on Nutrition concluded that the supplementation of infant formula with probiotics has an unclear effect on the consistency of the stool (35).

To observe the tolerance and compliance with the supplemented formula, we assessed the overall intake of formula. We found similar formula intake in both groups, and thus, the study results suggest that the $B$. infantis IM-1-supplemented formula is well accepted and safe in terms of infant compliance. Some authors have reported a similar intake between probiotic and control groups (25), whereas a recent meta-analysis showed that infants in the probiotic group had significantly higher formula intake than the control (15).

Some limitations of our study may be that some recruited infants had been fed breastmilk (11.2\%) or infant formula with pre- or probiotics (33.9\%). These infants underwent a washout period ( 7 days for formula-fed infants and 15 days for breastfed infants) and were equally distributed between the study groups, but we cannot reject that this previous feeding pattern may have influenced our results. Another potential source of bias is the use of antibiotics observed in $3.6 \%$ of the infants in our sample. Unfortunately, we cannot discard the use of antibiotics in some more infants because of incomplete data in the medical diary record. In total, an $85.8 \%$ of the infants did not receive any antibiotic during the study without differences in distribution between study groups $(P=0.255)$.

In summary, this study suggests that $B$. infantis IM-1 supplementation may prevent diarrhea episodes and is associated with a lower incidence of constipation as well as higher stool frequency compared with the control group. In addition, growth rate and digestive tolerance of the supplemented formula is adequate and similar to those of the nonsupplemented formula. We also found a relationship between the total IgA concentrations and the B. Infantis IM- 1 content in the fecal microbiota. Thus, we conclude that supplemented infant formula containing $10^{7} \mathrm{cfu} / \mathrm{g}$ of Bifidobacterium longum subsp. infantis CECT7210 (B. infantis IM-1) is safe, effective, and well tolerated in healthy term infants. 


\section{Articles | Escribano et al.}

\section{STATEMENT OF FINANCIAL SUPPORT}

This clinical trial was funded by Laboratorios ORDESA SL.

Disclosure: Mireia Morera, Jose Antonio Moreno Muñoz, and Montserrat Rivero are employees of ORDESA SL. Their role was to critically review the manuscript and were not involved in the statistical analysis or manuscript redaction. The remaining authors declare no conflict of interest.

\section{REFERENCES}

1. Matamoros S, Gras-Leguen C, Le Vacon F, et al. Development of intestinal microbiota in infants and its impact on health. Trends Microbiol 2013;21:167-73.

2. Gerritsen J, Smidt H, Rijkers GT, et al. Intestinal microbiota in human health and disease: the impact of probiotics. Genes Nutr 2011;6: $209-40$.

3. Martín R, Langa S, Reviriego C, et al. Human milk is a source of lactic acid bacteria for the infant gut. J Pediatr 2003;143:754-8.

4. Chen J, Cai W, Feng Y. Development of intestinal bifidobacteria and lactobacilli in breast-fed neonates. Clin Nutr 2007;26:559-66.

5. Harmsen HJ, Wildeboer-Veloo AC, Raangs GC, et al. Analysis of intestinal flora development in breast-fed and formula-fed infants by using molecular identification and detection methods.J Pediatr Gastroenterol Nutr 2007;30 (5): 61-7.

6. Lara-Villoslada F, Olivares M, Sierra S, et al. Beneficial effects of probiotic bacteria isolated from breast milk. Br J Nutr 2007;98:S96-100.

7. Oozeer R, van Limpt $\mathrm{K}$, Ludwig $\mathrm{T}$, et al. Intestinal microbiology in early life: specific prebiotics can have similar functionalities as human-milk oligosaccharides. Am J Clin Nutr 2013;98:561S-71S.

8. Saavedra JM, Abi-Hanna A, Moore N, et al. Long-term consumption of infant formulas containing live probiotic bacteria: tolerance and safety. Am J Clin Nutr 2004;79:261-7.

9. Committee E, Braegger ÃC, Chmielewska ÃA, et al. Supplementation of infant formula with probiotics and / or prebiotics: a systematic review and comment by the ESPGHAN committee on nutrition. J Pediatr Gastroenterol Nutr 2011;52:238-50.

10. Rautava S, Salminen S, Isolauri E.Specific probiotics in reducing the risk of acute infections in infancy-a randomised, double-blind, placebocontrolled studyBr J Nutr 2009;101 (11): 1722-6.

11. Maldonado J, Cañabate F, Sempere L, et al. Human milk probiotic Lactobacillus fermentum CECT5716 reduces the incidence of gastrointestinal and upper respiratory tract infections in infants. J Pediatr Gastroenterol Nutr 2012;54:55-61.

12. Osborn DA, Sinn JK.Prebiotics in infants for prevention of allergic disease and food hypersensitivityCochrane Database Syst Rev 2007 (1469-493X (Electronic)): CD006474.

13. Miele E, Pascarella F, Giannetti E, et al. Effect of a probiotic preparation (VSL\#3) on induction and maintenance of remission in children with ulcerative colitis. Am J Gastroenterol 2009;104:437-43.

14. Guarner F, Malagelada JR. Gut flora in health and disease. Lancet 2003;361:512-9.

15. Mugambi MN, Musekiwa A, Lombard M, et al. Synbiotics, probiotics or prebiotics in infant formula for full term infants: a systematic review.. Nutr J 2012;11:81.

16. Szajewska H, Guarino A, Hojsak I, et al. Use of probiotics for management of acute gastroenteritis : a position paper by the ESPGHAN Working Group for Probiotics and Prebiotics. J Pediatr Gastroenterol Nutr 2014;58:531-9.

17. Muñoz JA, Chenoll E, Casinos B, et al. Novel probiotic Bifidobacterium longum subsp. infantis CECT 7210 strain active against rotavirus infections. Appl Environ Microbiol 2011;77:8775-3.

18. Carrascosa Lezcano A, Ferrández Longás A, Yeste Fernández D, et al. Parte I: valores de peso y longitud en recién nacidos de 26-42 semanas de edad gestacional. An Pediatr 2008;68:544-1.

19. WHO WHO Anthro software for PC 2009.

20. Colomina J, Gil MT, Codoñer P, et al. Viral proteins VP2, VP6, and NSP2 are strongly precipitated by serum and fecal antibodies from children with rotavirus symptomatic infection. J Med Virol 1998;56:58-65.
21. Matsuki T, Watanabe K, Fujimoto J, et al. Use of $16 \mathrm{~S}$ rRNA genetargeted group-specific primers for real-time PCR analysis of predominant bacteria in human feces. Appl Environ Microbiol 2004;70:7220-8.

22. Yuan S, Cohen DB, Ravel J, et al. Evaluation of methods for the extraction and purification of DNA from the human microbiome. PLoS ONE 2012;7:e33865.

23. Sim K, Cox MJ, Wopereis $\mathrm{H}$, et al. Improved detection of bifidobacteria with optimised $16 \mathrm{~S}$ rRNA-gene based pyrosequencing. PLoS ONE 2012;7:e32543.

24. Caporaso JG, Kuczynski J, Stombaugh J, et al. QIIME allows analysis of high-throughput community sequencing data. Nat Methods 2010;7:335-6.

25. Weizman Z, Asli G, Alsheikh A. Effect of a probiotic infant formula on infections in child care centers: comparison of two probiotic agents. Pediatrics 2005;115:5-9.

26. Grześkowiak Ł, Grönlund M-M, Beckmann C, et al. The impact of perinatal probiotic intervention on gut microbiota: double-blind placebocontrolled trials in Finland and Germany. Anaerobe 2012;18:7-13.

27. Campeotto F, Suau A, Kapel N, et al. A fermented formula in pre-term infants: clinical tolerance, gut microbiota, down-regulation of faecal calprotectin and up-regulation of faecal secretory IgA. Br J Nutr 2011;105: 1843-51.

28. Mah KW, Chin VIL, Wong WS, et al. Effect of a milk formula containing probiotics on the fecal microbiota of asian infants at risk of atopic diseases. Pediatr Res 2007;62:674-9.

29. Di Gioia D, Aloisio I, Mazzola G, et al. Bifidobacteria: their impact on gut microbiota composition and their applications as probiotics in infants. Appl Microbiol Biotechnol 2014;98:563-77.

30. Gibson RA, Barclay D, Marshall H, et al. Safety of supplementing infant formula with long-chain polyunsaturated fatty acids and Bifidobacterium lactis in term infants: a randomised controlled trial. Br J Nutr 2009;101: 1706-3.

31. Holscher HD, Czerkies LA, Cekola P, et al. Bifidobacterium lactis Bb12 enhances intestinal antibody response in formula-fed infants: a randomized, double-blind, controlled trial. JPEN J Parenter Enteral Nutr 2012;36 (1 Suppl): 106S-7SS.

32. Nagao AT, Pilagallo M, Pereira AB, et al. Quantification of salivary, urinary and fecal secretory $\operatorname{IgA}$, as well as in saliva titers and avidities of IgA antibodies in children living at different levels of antigenic exposure and undernutrition. Adv Exp Med Biol 1995;371A:507-11.

33. Weizman Z, Alsheikh A. Safety and tolerance of a probiotic formula in early infancy comparing two probiotic agents: a pilot study. J Am Coll Nutr 2006;25:415-9.

34. Ziegler EE, Jeter JM, Drulis JM, et al. Formula with reduced content of improved, partially hydrolyzed protein and probiotics: infant growth and health. Monatsschrift Kinderheilkd 2003;151:S65-71.

35. Braegger C, Chmielewska A, Decsi T, et al. Supplementation of infant formula with probiotics and/or prebiotics: a systematic review and comment by the ESPGHAN committee on nutrition. J Pediatr Gastroenterol Nutr 2011;52:238-50.

36. Chouraqui J-P, Van Egroo L-D, Fichot M-C. Acidified milk formula supplemented with Bifidobacterium lactis: impact on infant diarrhea in residential care settings. J Pediatr Gastroenterol Nutr 2004;38:288-92.

37. Hyams JS, Treem WR, Etienne NL, et al. Effect of infant formula on stool characteristics of young infants. Pediatrics 1995;95 0031-4005 (Print) $50-4$.

38. Vendt N, Grünberg H, Tuure T, et al. Growth during the first 6 months of life in infants using formula enriched with Lactobacillus rhamnosus GG: double-blind, randomized trial. J Hum Nutr Diet 2006;19:51-8.

39. Tabbers MM, DiLorenzo C, Berger MY, et al. Evaluation and treatment of functional constipation in infants and children: evidence-based recommendations from ESPGHAN and NASPGHAN. J Pediatr Gastroenterol Nutr 2014;58:258-74.

40. Petschow BW, Figueroa R, Harris CL, et al. Effects of feeding an infant formula containing Lactobacillus GG on the colonization of the intestine: a dose-response study in healthy infants. J Clin Gastroenterol 2005;39: 786-90. 